\title{
Payload Data Processing System on Satellite
}

\author{
Shreyas H V ${ }^{1}$, Dr Mohan H S ${ }^{2}$ \\ IV Semester, M Tech Student, CNE Department, SJBIT, Bangalore, India ${ }^{1}$ \\ Professor and Head, Department of ISE, SJBIT, Bangalore, India ${ }^{2}$
}

\begin{abstract}
The world is progressively relying upon theremotely detected information. The information is routinely utilized for checking the earth assets and furthermore to solve issues of the world like fiascos, atmosphere debasement, and so forth. Remotely detected information has changed our point of view of comprehension of different planets. With imaginative methodologies in information use, the requests of remote detecting information are regularly expanding. New exploration and advancements are obtained from information use. Satellite assets remain rare and each dispatch outlays vigorously. Apiece dispatch is likewise connected with expansive exertion for building up the equipment preceding dispatch. It is additionally connected with a substantial number of programming components and scientific calculations post-dispatch. The multiplication of low-earth and geostationary satellites has prompted expanded shortage in the accessible orbital spaces for the more up to date satellites. Space Research Organization has constantly attempted to upturn the service of satellites. Different sensors are winged on each satellite. In a piece of the satellites, radars are intended to take into account different ghostly groups/rates. Satellite (ex-Bhaskara-1), the principal trial satellite began with two groups in electro-optical range and three groups in themicrowave range. The framework stood intended to give information all around different information gathering stations and locally available information stockpiling abilities. Oceansat-2 satellite has one of a kind sensor mix with 8 bands electro-optical high delicate sea shading screen (obliging sea and land) alongside Ku-band scatter meter to procure data on sea winds. A similar framework has information transfer transponders for gaining information from climate stations. The payload arrangements have experienced noteworthy changes throughout the existences to build information of payload. Upcoming Indian distant detecting frameworks are arranged with high proficient methods for picture procurement. This paper explains about the high effectiveness in remote detecting picture information obtaining. Parameters identified with the effectiveness of picture information procurement are characterized then a philosophy is operated out to register the identical. A portion of the payloadsis broke down as for a portion of the framework limits that choose the arrangement of payload. This strategy might be utilized for surveying missions and can be reached out near different sorts of payloads and missions.
\end{abstract}

Keywords: Remote Sensing, Image acquisition, Resolution, Electro-optical payload, Data acquisition system, image acquisition efficiency.

\section{INTRODUCTION}

The dispatch of first satellite opened up open doors for space investigation. There have been changes in view how we see our planet by the help of the satellite which has remote sensing in them there has been a lot of development has been going on upcoming satellite. There has been aneffort to maintain the satellite movement and organize different type of satellite as coordination to aground station which they keep the track of each movement.

Today, satellites and their advantages are superior. Eventually, there are around 2,500 working satellites, of which 75\% are rubbish. Out of effective outposts, half are in Low-Earth Orbit. Further satellites are depended upon to be moved in future. Both Government and private Organizations from Forty-onecountries will dispatch many satellites by 2023. In light of conveyed report (3), 1155 satellites (Earth recognition and Communications) will be impelled all through the next decade (2014-2023). LEO Earth observation (EO) satellites will Increase to 353 appeared differently in relation to 162 more than 2004-2013.

Remote detecting satellites are checked by an arrangement of telemetry, following and order operation. These summon operations are helped out from any of the ground stations, which are obvious to the satellite. There are many ground stations in the system for the season of deceivability time is known as the breath easy, the ground station gets information from the satellite. This paper deals with theapproach of analyzing the appearance of payload and satellite. As a part of this payload structures of approximately sensing satellite which haselectro-optical instrument.

\section{PAYLOADMETHODS ARCHITECTURES}

Traditional Involved payload information frameworks have fundamentally three capacities:

- Gathering information cricks delivered by devices and gadgets 
- Transitory capacity of the obtained information to cross over any barrier between progressive earth position periods

○ Configuring of the link information creek for earth broadcast

The design of a framework is comparing to a pipeline. In past missions, the mass memory capacity was given by delicate recording devices favorably nowadays by strong formal corpus recollections.

Considering the growing data rates which have formed new generations of tools for earth observation and science mission, more functionality have been introduced for the payload data system the solution was to introduce the facts handling in order to decrease the data volume which has been transmitted

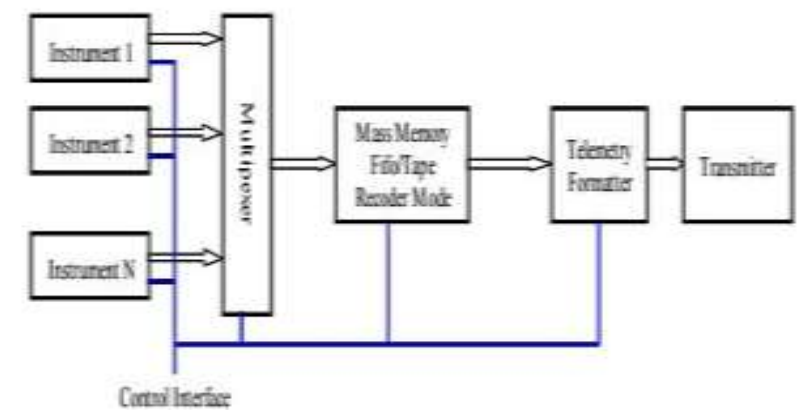

Figure1: synchronous pipeline architecture

From the above figure case onboard of system consists

○ In the data filtering data processing inherently reduces the amount of data

- In the data firmness, the data link can be decreased to low level

- In the data selection, the factors resultant will select data for ground mission

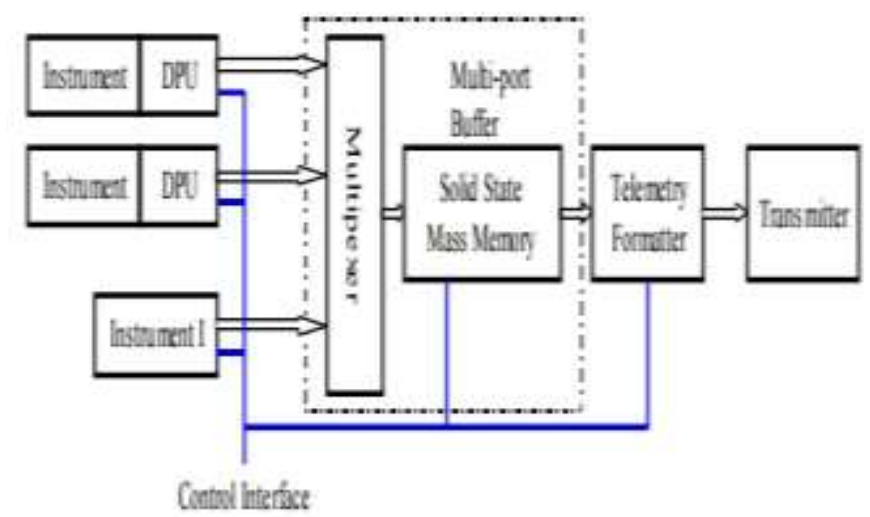

Figure 2: pipeline architecture with local embedded Digital processing unit

From the figure 2, we represent the digital processing unit to instruments for the onboard processing .most of earth satellites all which has been launched All the payload method still grieves from a lack of elasticity and failure retrieval capability. If a digital handling unit fails it cannot be replaced by another DPU. From the above architecture, we have alot of drawbacks because it persuades extended and expensive increase and manufacturing phases.

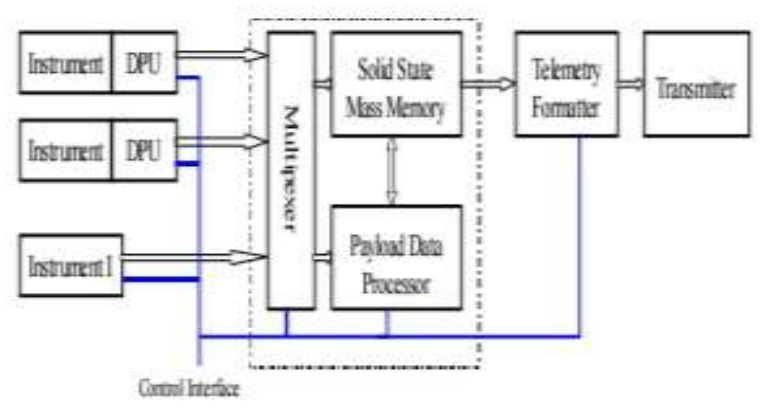

Figure 3: payload data system with intelligent mass memory 
And also second architecture deals with a lot of cost of the mock track. In nearcircumstances, there is a duplication of activities that and function and excessive mass and power budgets.

From the above figure 3, we have the advanced revolutionary advances provided by the instance by simulation and modeling .consequently in some cases a faster prototype tool has been with the system

\section{SPACE WIRING ROUTING SWITCHES}

A Space Wire coordinating switch relates together numerous centers and gives techniques for controlling bundles from one center point to one of numerous other possible center points. A Space Wire guiding switch contains different Space Wire associated interfaces and a coordinating structure. The controlling system enables packages meeting up at one association line to be traded and passed on to additional association line on coordinating switch. Every association interface might be measured as containing an information port (the association interface recipient) and a yield port (the association interface source).

A Space Wire coordinating switch trades packages afterthe data port of the switch where the package meets up, to a particular port controlled by package objective.

$\checkmark \quad$ Y path addressing: Way having a tendency to is used to indicate the course through a framework specifically.

$\checkmark \quad \mathrm{Y}$ logical addressing: Coherent Tending to is used to determine the course through a framework in a roundabout way by methods for coordinating stands seized in the regulatory switches.

\section{TOOLS AND PARALLEL DEVELOPMENTS}

Gadgets make sure it has not been disregarded and actions are being impelled to make and have showcased EGSE interface sheets and framework checking instruments. Two distinct exercises have endeavored. The past regroups under the "Top Net" action a thought allowing basic and serious decentralized structure coordination through heterogeneous frameworks, i.e. Space Wire, Intranets, and the Web.

A lot of tools has been introduced and changes have been made for the payload in all satellite stations.A lot of failures has happened and alot of changes have been made in gadget because of lot of good data were received from thesatellite and was obtain the successful.

\section{IMPLEMENTATION}

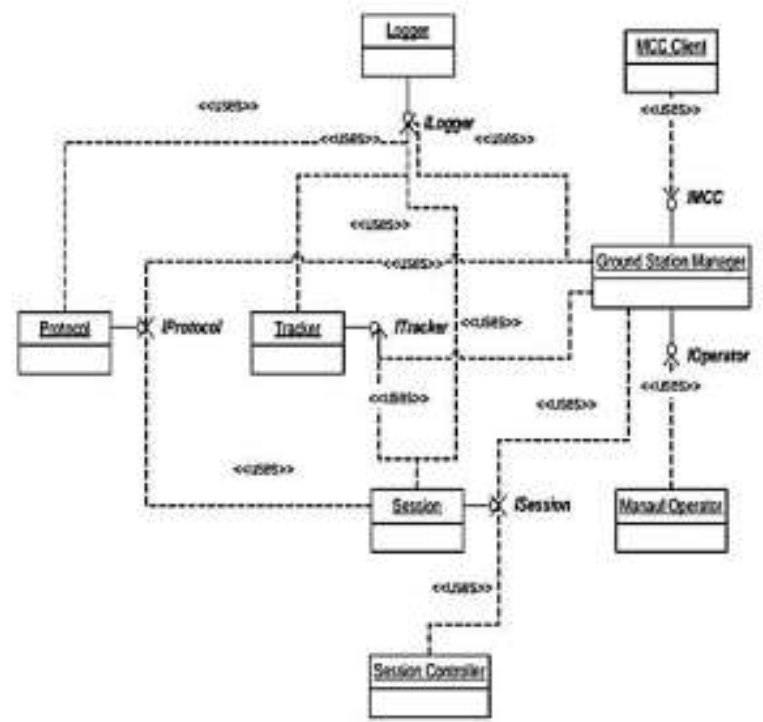

Fig 3.1: Interface design

Interfaces control the things in a machine. A class can implement numerous interfaces; however, it just can make greater one superclass to acquire the factors and methods

There will plan developed via the project control center $(\mathrm{mcc})$ and dispatched to the floor station manager. So the consultation have to have a few crucial residences identified with correspondence with satellites, for example, the begin time, end time, satellite call

In the application design, we give the administration to run to start a session and implement a session 


\section{PROTOCOL DESIGN}

\begin{tabular}{|l|}
\hline \multicolumn{1}{|c|}{$\begin{array}{l}\text { < } \\
\text { IProtocolace }\end{array}$} \\
\hline +startProtocol() \\
+stopProtocol() \\
+getStatus() \\
+connectTo()
\end{tabular}

Figure 3.1.1: protocol design

In this, we have protocol such as start protocol and stop protocol which has been helped in starting of protocol and stops when there is fatal error andgets status can help us to get update from the mission control

\section{RESULT AND DISCUSSION}

Figures 4.1 to 4.5 exhibit capability parameters $4 \mathrm{E}-1$ to $4 \mathrm{E}-6$ of some of these missions. It can be seen from Figures 4.1 to 4.5 that the viability parameters upgraded by and large over the missions. $4 \mathrm{E}-1$ extended from 9 to $100000 \mathrm{Kbps} / \mathrm{Kg}$ (four solicitations), 4E-2 from 18 to $53000 \mathrm{Kbps} / \mathrm{W}$ (> 3 orders), 4E-3 <1 to 96000 Phantom bits/W (5 orders), 4E-4 from $<1$ to 48000 spectral bits/Kg (5 solicitations) and $4 \mathrm{E}-5$ extended to $4.3 \mathrm{~GB} / \mathrm{Kg} / \mathrm{day}$ and $4 \mathrm{E} 6$ extended to $3 \mathrm{~GB} / \mathrm{W} /$ day, this are the performance based on Indian satellite

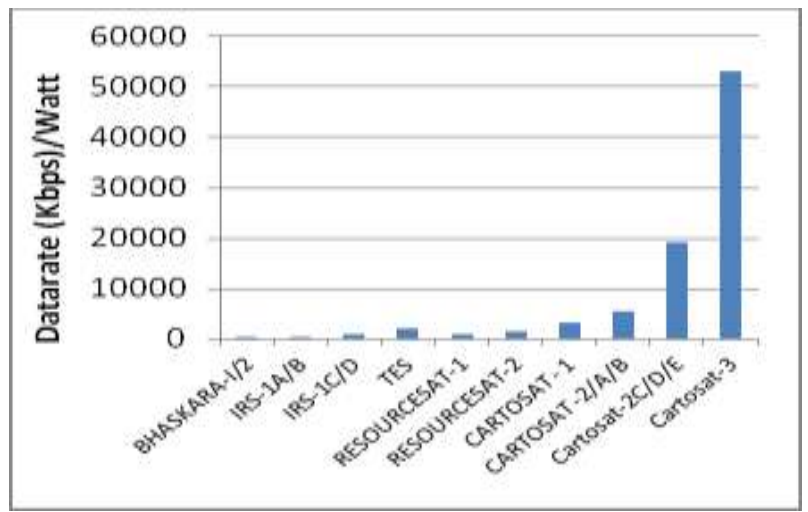

Figure4.1: 4E1

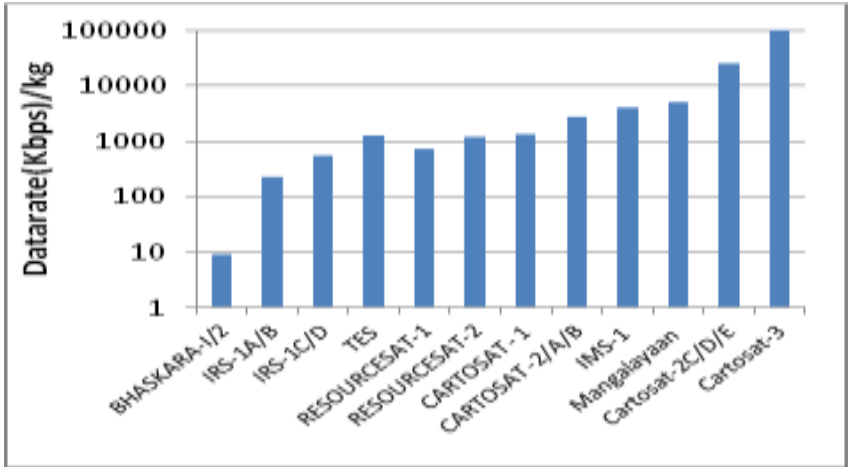

Figure 4.2: 4E2

Advanced payload setup a contextual investigation:

It can be told from the above investigation

- Extensive number of phantom diverts in a solitary

- High three-dimensional

- Minimal and lightweight optics 


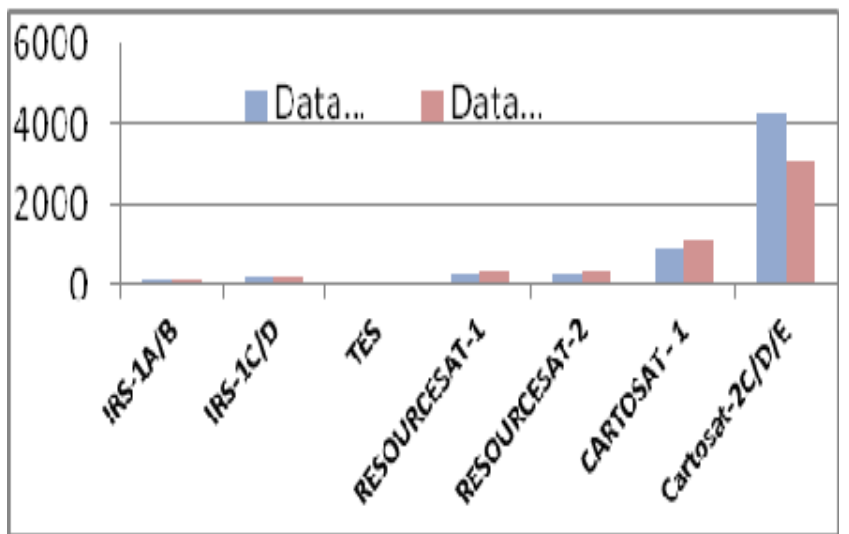

Figure 4.3:4E3

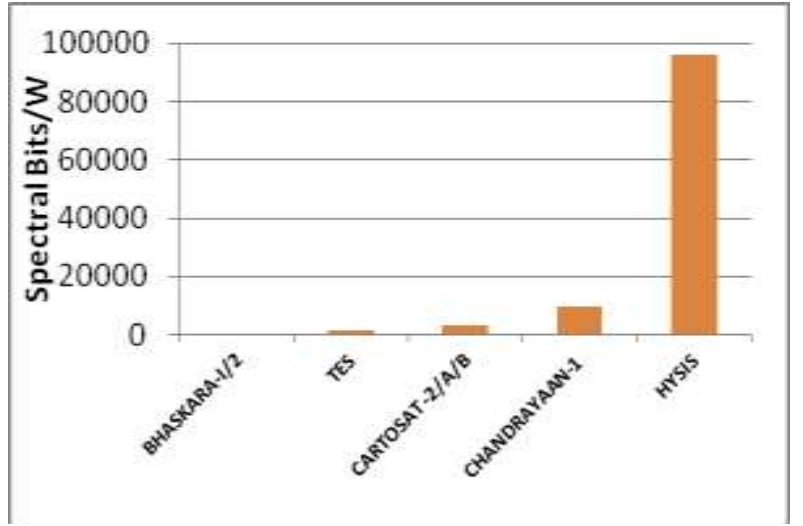

Figure 4.4:4E4

Payload Information Preparing systems have created from basically synchronous pipelines to more versatile models.

\section{CONCLUSION}

In this paper, points of view connected to the capability of the imaging system are discussed. The profitability limits critical for data/picture acquirements are described as a technique was created to get in contact at these qualities for a bit of the payload and missions using structure level parameters. An examination of these parameters is furthermore finished using the payload's remote distinguishing parameters and plan parameters. The examination exhibits that the viability improves basically over the missions on account of the expansion of apparition occupies in a given optical head, increase in spatial, supernatural and radiometric resolutions, usage of littler, low mass and low power optical, electro-optical, mechanical and electronic structures.

\section{ACKNOWLEDGEMENT}

Many developments have been necessary for the development of paper special thanks to Dr. Mohan HS, Professor, and Head, Department of ISE, SJBIT in providing the support in developing the paper.

\section{REFERENCES}

[1] “Authenticated Key Exchange Protocol for Parallel Network File System", Hoon Wei Lim, Guomin Yang, Parallel and Distributed Systems volume: 27, ISSUE: 1,2016

[2] "Innovation in cloud computing: Implementation of Kerberos version5in cloud computing inorder to enhance security issues", Hojabri, M.,Rao,K.V. Information Communication and Embedded Systems (ICICLES), Pages: 452 - 456, 2013.

[3] "An Anti-phishing Framework using Visual Cryptography", Abhishek Thorat, Mahesh More, Ganesh Thombre,International Journal of Advanced Research in Computer and Communication Engineering, Vol. 4, Issue 2, 2015.

[4] "Two-factor Authentication using Visual Cryptography and Digital Envelope in Kerberos", Khandelwal, N.S., Kamboj.P electrical, Electronics, Signals, Communication and Optimization (ESCO), 2015,Pages: 1 - 6.

[5] "Key management for large scale storage distributed Storage Systems",Hoonwelim,SPA Sophia Antipolis research, France.

[6] "An extended review of visual cryptography schemes", Ramya.J, Parvathavarthini.B, Control, Instrumentation Communication and Computational Technologies 